\title{
Enhancement of the magnetization saturation in magnetite (100) epitaxial films by thermo-chemical treatment
}

\author{
Y. Zhou, Xuesong Jin, and I. V. Shvets \\ SFI Nanoscience Laboratory, Trinity College, Dublin, Dublin 2, Ireland
}

(Presented on 9 January 2004)

\begin{abstract}
The effect of thermo-chemical treatment on the ease of saturation in a magnetic field of epitaxial magnetite (100) thin films grown on $\mathrm{MgO}$ (100) substrates was investigated. It was found that the films maintained a fully strained state with the $\mathrm{MgO}$ substrate during the treatment in air. No other iron oxide phase apart from the magnetite was observed in the treated film. Yet, remarkably the treated films showed a higher magnetization compared to that of original ones. A model involving a change in antiferromagnetic coupling at the antiphase boundaries during the treatment is proposed. (C) 2004 American Institute of Physics. [DOI: 10.1063/1.1687632]
\end{abstract}

\section{INTRODUCTION}

Epitaxial films of spinels are of scientific and technological importance. Magnetite $\left(\mathrm{Fe}_{3} \mathrm{O}_{4}\right)$ is a half-metallic ferromagnet spinel material and as a result it currently attracts a lot of interest from the spin electronics community. Much emphasis has been placed on the growth of $\mathrm{Fe}_{3} \mathrm{O}_{4}$ singlecrystalline films by various deposition methods using $\mathrm{MgO}$, $\mathrm{Si}, \mathrm{Al}_{2} \mathrm{O}_{3}$, and $\mathrm{MgAl}_{2} \mathrm{O}_{4}$ as a substrate. It was reported that antiphase boundaries (APBs) exist in epitaxial magnetite films. ${ }^{1-3}$ In the case of the growth of magnetite on the most common substrate, $\mathrm{MgO}$, the APBs arise from the fact that the lattice constant of magnetite is twice that of the substrate resulting in half-lattice period shifts between some of the nucleation islands. Antiferromagnetic exchange through the APBs makes saturating this soft magnetic material difficult. ${ }^{4,5}$ As a result the potential for applications of magnetite films in spin electronic devices is hampered.

This study addresses the following question: is it possible to treat magnetite so that it can then be more easily saturated by an external field? $\mathrm{Fe}_{3} \mathrm{O}_{4}$ (100) thin films grown on $\mathrm{MgO}(100)$ substrates using oxygen-plasma-assisted molecular beam epitaxy were used for this study. The effect of the thermo-chemical treatment process on the ease of saturation in a magnetic field was investigated.

\section{EXPERIMENTAL DETAILS}

Epitaxial magnetite thin films with a thickness of 100 $\mathrm{nm}$ were grown on $\mathrm{MgO}$ single-crystal substrates cut along the $\langle 100\rangle$ direction within $\pm 0.1^{\circ}$ by oxygen-plasma-assisted molecular beam epitaxy. The base pressure in the growth chamber was lower than $5 \times 10^{-10}$ Torr. Each MgO substrate was annealed at $600{ }^{\circ} \mathrm{C}$ for $30 \mathrm{~min}$ in a plasma oxygen environment prior to deposition. The magnetite layer was deposited by means of $e$-gun evaporation from Fe pellets with a purity of $99.995 \%$ in a plasma oxygen environment of $1 \times 10^{-5}$ Torr with a substrate temperature of $250{ }^{\circ} \mathrm{C}$. Growth mode and crystalline quality of the films were monitored in situ by reflection high-energy electron diffraction (RHEED). Film thickness was controlled by quartz-crystal thickness monitors calibrated using X-ray reflectivity.
A BEDE D1 diffractometer was employed to carry out the high resolution $\mathrm{X}$-ray diffraction (HRXRD) measurements. The diffractometer was equipped with a channel cut Si crystal monochromator. It was further equipped with a TTK 450 Anton Paar chamber. This facility allowed the samples to be annealed in situ without removing them from the HRXRD setup. Samples were annealed in air at $250{ }^{\circ} \mathrm{C}$. The detailed annealing and HRXRD measurement procedure is described in Ref. 6. The position of the diffraction peaks and a reciprocal space map (RSM) were employed to evaluate the strain status of the epitaxial films during the treatment. Raman spectroscopy (RS) measurements were carried out before and after the treatment to clarify the treatment induced phase on the magnetite surface. The four-probe method was employed in the resistance measurements. Hysteresis loop and magnetic moment measurements were carried out using a MicroMag 3900 alternating gradient force magnetometer at room temperature. To avoid the error introduced by the distribution of film thickness and volume among different film specimens, single specimen was employed for magnetic measurement before and after the treatment.

\section{RESULTS AND DISCUSSION}

The RHEED results suggest that the magnetite films were grown epitaxially as confirmed by the observed oscillations of pattern intensity. A resistivity jump was observed around $115 \mathrm{~K}$ indicating that the films were substantially stoichiometric. The somewhat lower observed Verwey transition temperature of films compared to values for single crystals $(120 \mathrm{~K})$ is in agreement with previous reports on magnetite films. ${ }^{7}$ RS measurements which were carried out on original and treated films suggest that there are no traces of other iron oxide phases apart from magnetite in the original films as well as films annealed at $250{ }^{\circ} \mathrm{C}$ for up to 160 min. The out-of-plane and in-plane lattice parameters of the original film as calculated from the HRXRD results were 8.3604 and $8.4268 \AA$, respectively. The in-plane lattice parameter of the film is twice that of the $\mathrm{MgO}$ substrate implying that the film is fully strained on the $\mathrm{MgO}$ substrate. The 


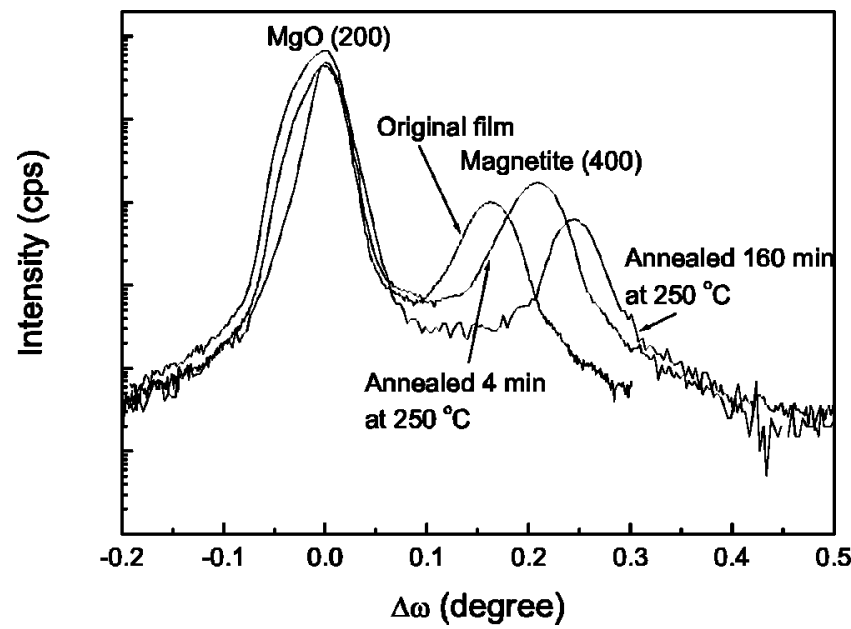

FIG. 1. Rocking curve of the magnetite film of symmetric (400) diffraction before and after treatment.

(400) symmetric rocking curves of the original and treated films are shown in Fig. 1. The diffraction peak does shift to the higher angle side with annealing in air. To establish if the shift is due to the relaxation of the film strain or a change in the volume of the unit cell, we have performed RSM measurements near the asymmetric peaks. RSM near the asymmetric (226) reflection of the original and treated films were obtained by carrying out several $\omega-2 \theta$ scans for different values of $\omega$. A contour plot of the intensity as a function of the in-plane and the out-of-plane reciprocal wave vectors $k_{\|}$ and $k_{\perp}$ for a film annealed for $160 \mathrm{~min}$ is shown in Fig. 2. $k_{\|}$ of the magnetite film is equal to that of the $\mathrm{MgO}$ substrate which indicates that the in-plane lattice parameter of the substrate and film are equal. Our results suggest that the films remain in a fully strained state after the treatment. Therefore, the observed (400) peak shift after a long annealing time results from decrease of lattice parameter perpendicular to the surface and a subsequent decrease in the volume of the unit cell. The change in the stoichiometry of the treated films was also evaluated from $R-T$ curves (see Fig. 3). Verwey transitions that are suppressed even by small deviations from

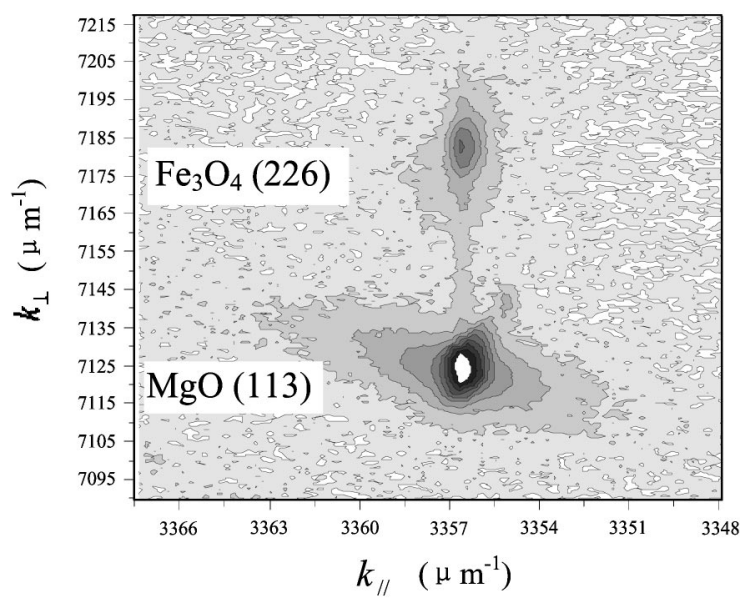

FIG. 2. Reciprocal space map near asymmetric (226) glancing exit diffraction of a magnetite film annealed in air at $250^{\circ} \mathrm{C}$ for $160 \mathrm{~min}$.

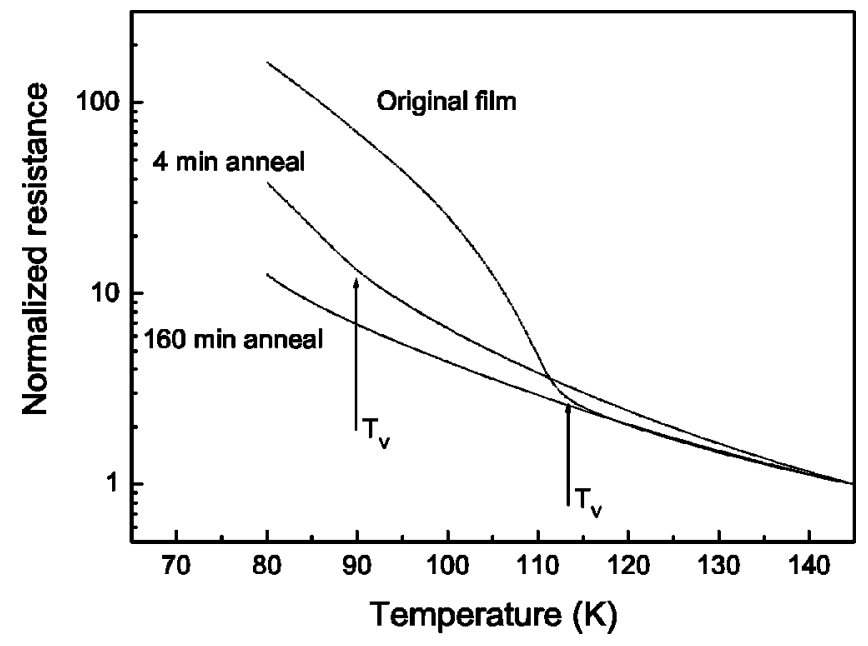

FIG. 3. Resistances of original and treated magnetite films vs temperature normalized to their respective resistance values at $150 \mathrm{~K}$.

stoichiometry ${ }^{8}$ can still be observed in films subjected to 4 min annealing suggesting that their stoichiometry remained close to that of ideal $\mathrm{Fe}_{3} \mathrm{O}_{4}$. One can therefore arrive at the conclusion: annealing in air at $250^{\circ} \mathrm{C}$ for 4 min does not significantly change the stoichiometry of the film. The magnetization and coercivity dependencies on applied field for the original and treated films are shown in Fig. 4(a). The dependency of magnetic moment on an external magnetic field of strength $1 \mathrm{~T}\left(M_{1 \mathrm{~T}}\right)$ and coercivity of the magnetite film on annealing time are shown in Fig. 4(b). Very short annealing significantly decreases the coercivity. What is more remarkable is that the film after a short annealing in air becomes much easier to saturate. $M_{1 \mathrm{~T}}$ manifests itself as a substantial increase in the value of saturation magnetization $M_{\mathrm{S}}$ caused by short treatment. Before discussing the possible physical mechanisms responsible for the observed results, we must first consider the effect of a change in the oxygen stoichiometry of the films induced by the treatment on their magnetic properties. It is known that a significant change in $X$ in $\mathrm{Fe}_{3-X} \mathrm{O}_{4}$ from 0.015 to 0 leads to a change in $M_{\mathrm{s}}$ of only $1 \% .^{9}$ As established from HRXRD, RS, and resistivity measurements, the change in the film's stoichiometry caused by annealing in air was marginal. Such a small change cannot explain a massive $14 \%$ increase observed in the value of $M_{1 \mathrm{~T}}$. It is expected that the $M_{\mathrm{s}}$ of films will decrease upon oxidation from ideal stoichiometry state. Furthermore, $M_{\mathrm{s}}$ of the films is expected to change incrementally with increasing anneal time. However, the dependency of $M_{1 \mathrm{~T}}$ on the anneal time does not favor such an assumption of a gradual stoichiometry change. We observed that the greatest increase in the value of $M_{1 \mathrm{~T}}$ is caused by initial short annealing.

Our explanation for the significant increase in the magnetization is based on the exchange interaction at the antiphase boundaries. It is clear from the body of the publications on the magnetization of magnetite epitaxial films, the reduced magnetization results from antiferromagnetic and frustrated exchange interaction across APBs. It is rather difficult to come up with a more specific model as there are many contributions to the exchange interaction. For example, Margulies et al. suggested that at APBs antiferromagnetic 

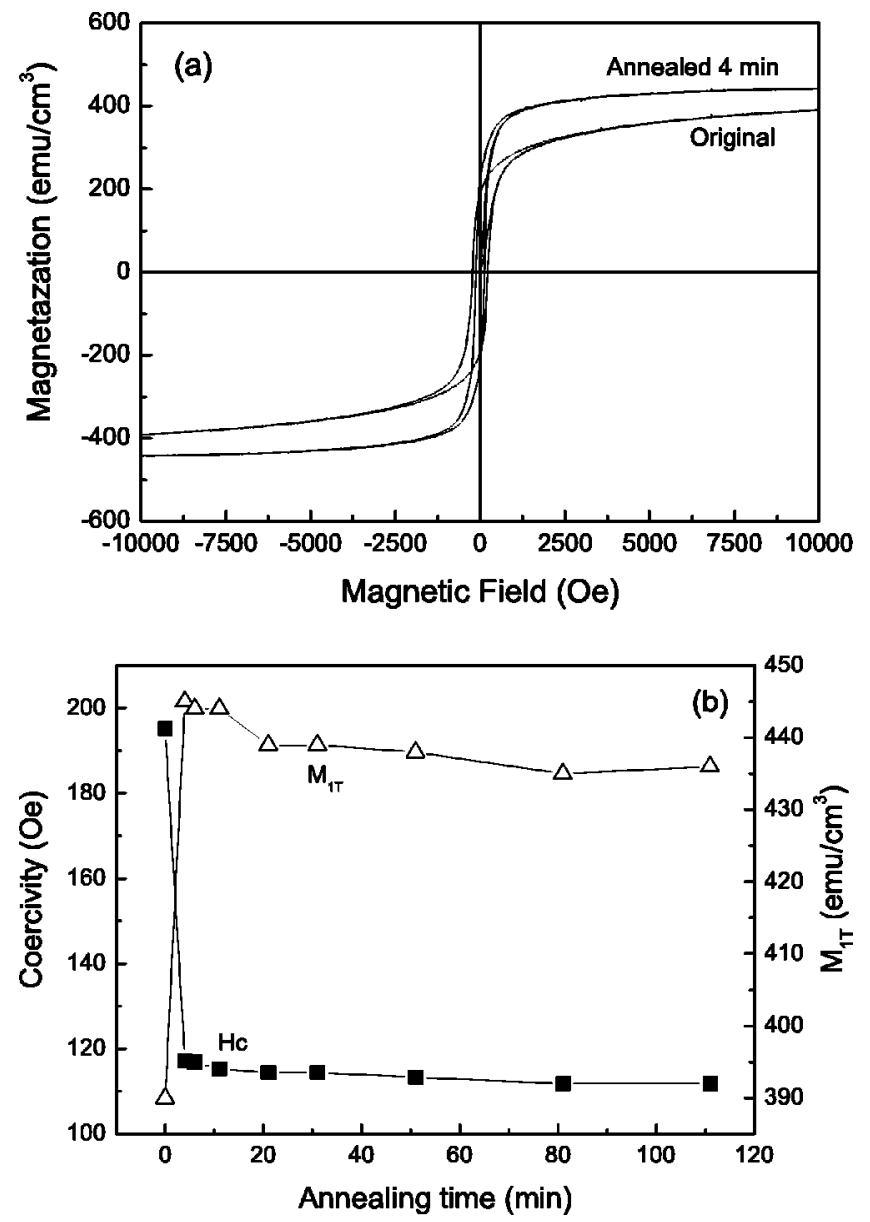

FIG. 4. (a) Hysteresis loop of original magnetite film and the one annealed in air for $4 \mathrm{~min}$. (b) The dependency of magnetic moment at $1 \mathrm{~T}$ external magnetic field and coercivity of magnetite film on time of annealing in air.

interaction between atoms of A sublattice (tetrahedral interstices) atoms is greatly increased, a very strong antiferromagnetic interaction between cations on the B sublattice (octahedral interstices) is created, and the normally dominant $\mathrm{AB}$ interaction is greatly reduced. ${ }^{10}$ The establishment of the atomic structure of the APBs is necessary to fully understand their magnetic behavior. What is beyond doubt, however, is that the stoichiometry of the antiphase boundary is crucial to the exchange interaction across it. Indeed, most of the exchange interactions are $\mathrm{Fe}-\mathrm{O}-\mathrm{Fe}$ superexchange ones. Not only the removal or addition of anions to the antiphase boundary can modify the exchange interaction but also the rearrangement of atoms within the boundary. The reason is that superexchange for $180^{\circ}$ and $90^{\circ}$ chains of Fe-O-Fe ions can differ. What is also intuitively clear is that the stoichiometry of the APBs is more likely to change rather than that of the film area remote from it. Indeed, APBs are a metastable defect as opposed to a stable crystal structure. Our results therefore suggest that the increase in the value of the magnetization comes from the decay of the antiferromagnetic and frustrated exchange interaction that takes place through localized thermo-chemical reaction at the APBs or in their vicinity.

\section{CONCLUSION}

The effect of low temperature annealing in air on the magnetization and coercivity of epitaxial $\mathrm{Fe}_{3} \mathrm{O}_{4}$ (100) thin films was investigated. The as-grown films are fully strained on the $\mathrm{MgO}(100)$ substrate and remain in a fully strained state following the treatment. The treated film is significantly easier to saturate compared with the as-grown one. The magnetic moment increases remarkably following a short annealing in air of just 4 min, which does not greatly affect the film's composition. A model involving a change in the antiferromagnetic and frustrated exchange coupling at the APBs associated with the treatment is proposed.

\section{ACKNOWLEDGMENT}

This work was supported by Science Foundation Ireland (SFI) under the contract No. 00/PI.1/C042.

${ }^{1}$ D. T. Margulies, F. T. Parker, and A. E. Berkowitz, J. Appl. Phys. 75, 6097 (1994).

${ }^{2}$ V. S. Speriosu, M. M. Chen, and T. Suzuki, IEEE Trans. Magn. 25, 3875 (1989).

${ }^{3}$ T. Fujii, M. Takano, R. Katano, and Y. Bando, J. Cryst. Growth 99, 606 (1990).

${ }^{4}$ D. T. Margulies, F. T. Parker, F. E. Spada, R. S. Goldman, J. Li, R. Sinclair, and A. E. Berkowitz, Phys. Rev. B 53, 9175 (1996).

${ }^{5}$ W. L. Zhou, K.-Y. Wang, C. J. O'Conner, and J. Tang, J. Appl. Phys. 89, 7398 (2001).

${ }^{6}$ Y. Zhou, Xuesong Jin, Y. M. Mukovskii, and I. V. Shvets, J. Phys.: Condens. Matter 16, 1 (2004)

${ }^{7}$ S. B. Ogale, K. Ghosh, R. P. Sharma, R. L. Greene, R. Ramesh, and T. Venkatesan, Phys. Rev. B 57, 7823 (1998).

${ }^{8}$ J. P. Shepherd, R. Aragon, J. W. Koenitzer, and J. M. Honig, Phys. Rev. B 32, 1818 (1985).

${ }^{9}$ Z. Kakol and J. M. Honig, Phys. Rev. B 40, 9090 (1989).

${ }^{10}$ D. T. Margulies, F. T. Parker, M. L. Rudee, F. E. Spada, J. N. Chapman, P. R. Aitchison, and A. E. Berkowitz, Phys. Rev. Lett. 79, 5162 (1997). 